AperTO - Archivio Istituzionale Open Access dell'Università di Torino

\title{
A unisex stochastic mortality model to comply with EU Gender Directive
}

\section{This is a pre print version of the following article:}

Original Citation:

\section{Availability:}

This version is available http://hdl.handle.net/2318/1633916

since 2017-05-15T11:43:50Z

Published version:

DOI:https://doi.org/10.1016/j.insmatheco.2017.01.007

Terms of use:

Open Access

Anyone can freely access the full text of works made available as "Open Access". Works made available under a Creative Commons license can be used according to the terms and conditions of said license. Use of all other works requires consent of the right holder (author or publisher) if not exempted from copyright protection by the applicable law. 


\title{
A unisex stochastic mortality model to comply with EU Gender Directive*
}

\author{
An Chen ${ }^{\dagger} \quad$ Elena Vigna ${ }^{\ddagger}$
}

January 24, 2017

\begin{abstract}
EU Gender Directive ruled out discrimination against gender in charging premium for insurance products. This prohibition prevents the use of the standard actuarial fairness principle to price life insurance products. According to current actuarial practice, unisex premiums are calculated with a simple weighting rule of the gender-specific life tables. This procedure is likely to violate portfolio fairness principles. Up to our knowledge, in the actuarial literature there is no unisex mortality model that respects the unisex fairness principle. This paper is the first attempt to fill this gap. First, we recall the notion of unisex fairness principle and the corresponding unisex fair premium. Then, we provide a unisex stochastic mortality model for the mortality intensity that is underlying the pricing of a life portfolio of females and males belonging to the same cohort. Finally, we calibrate the unisex mortality model using the unisex fairness principle. We find that the weighting coefficient between the males' and females' own mortalities depends mainly on the quote of portfolio relative to each gender, on the
\end{abstract}

*We thank the participants to the 19th International Congress on Insurance: Mathematics and Economics and those to the 3rd European Actuarial Journal Conference for useful remarks. We are indebted to Paolo De Angelis, Kristian Buchardt, Montserrat Guillen, Elisa Luciano, Pietro Millossovich, Luca Regis and two anonymous referees for very valuable comments that improved the paper.

${ }^{\dagger}$ Department of Mathematics and Economics, University of Ulm. Email: an.chen@uni-ulm.de.

${ }_{\ddagger}^{\ddagger}$ Corresponding author. University of Torino, Collegio Carlo Alberto and CeRP, Italy. Email: elena.vigna@unito.it. 
age, and on the type of insurance product. The knowledge of a proper unisex mortality model could help life insurance companies to better understanding the nature of the risk of a mixed portfolio.

Keywords. Actuarial fairness, unisex tariff, stochastic mortality intensity, Gender Directive, life table, doubly stochastic process.

JEL classification: C1, C13, C18, C38, J11.

\section{Introduction and motivation}

On 1 March 2011 the European Court of Justice ruled that differences in insurance premiums based on the policyholder's gender are discriminatory (European Union Directive 2004/113/EC, also called Gender Directive), and that gender equality in the European Union must be ensured from 21 December 2012. Therefore, insurance policies issued from 21 December 2012 cannot be priced according to the insured's gender. Life insurance policies pay benefits that depend on the duration of life of the insured, i.e. benefits are paid in case of occurrence of an event such as death or survivorship at a certain date or within a given time frame. According to the actuarial equivalence principle, the premium of an insurance policy is based on the probability that the event will occur, i.e., it depends on the probability that the insured will die or survive within a given time frame. It is well known that the death and survival probabilities of an individual are strongly related to his/her gender: women are more likely than men to survive at every given future date, everything else (age, health status etc.) being equal. The use of gender-specific mortality tables permits the calculation of actuarially fair premiums of insurance policies. The prohibition of using gender-specific price rates since December 2012 increases the difficulty in respecting actuarial fairness and in reserving, and affects competitiveness.

Depending on what type of insurances policies, policyholders' age and policy duration, the Gender Directive has both economic and actuarial implications. For the economic aspect, for instance [12] analyzes the effects of mandatory unisex tariffs on the optimal insurance 
demand and point out that it has an adverse effect on the insurer's portfolio. They show that unisex tariffs might cause market distortion, hence reduce the overall social welfare. [13] discusses unisex insurance pricing from the insurance industry's, the regulator's and ethical point of view. More importantly, through an international consumer survey conducted in the UK, Germany, France, Italy and Switzerland, they assess the customer's acceptance of price differentiation for diverse insurance products. [15] discusses also the fairness and equality in actuarial risk selection, but from a legal standpoint. They clarify the conditions for reaching a fair insurance-differentiation scheme. For the actuarial aspect, [5] suggests that gender information shall be taken into consideration when analyzing the insurance companies' data, despite the ban on the gender discrimination. In the future, other indicators like lifestyle information can serve as a better predictor than gender in the actuarial modelling, if insurance companies can pay more attention to data quality rather than data quantity. [6] models the general population in Mexico and compare it with Mexican unisex life tables that are used for insurance purposes. They suggest that unisex tariffs should be based on reasonable assumptions about the proportions of males and females in the mixed portfolio. They discuss the possible bias resulting from using the unisex life tables, particularly when the proportion of the male and female policyholders is not balanced.

A relevant problem that insurers have to deal with when charging unisex premiums is adverse selection. The insureds of one sex ("good risk" for the insurance company) have to pay a higher premium than before the mandatory unisex tariff; and the insureds of the other sex ("bad risk" for the insurance company) pay a lower premium than before the mandatory unisex tariff. This asymmetry might induce insureds with "good risk" to refrain from purchasing insurance, and insureds with "bad risk" to buy more insurance. Adverse selection might cause market distortions, which could be severe if (in the extreme case) eventually the portfolio consists of only insureds with "bad risk". In this case, the individual with "good risk", who does not find it convenient to buy the insurance product, may well feel discriminated, and one may even wonder whether the EU Gender Directive, which was introduced to prevent gender discrimination, has rather introduced gender discrimination in the insurance market. These important issues are beyond the scope of this paper. 
In this work we focus only on the actuarial aspects of the Gender Directive. An important issue is: What is the best way to reach actuarial fairness and respect the Gender Directive? The actuarial literature on this topic is silent. The current practice in insurance companies seems to be the calculation of a unisex tariff obtained by mixing the life tables of the two sexes, with a correction that takes into account the insurance product considered and gives more weight to the life table of the prevailing-risk gender. With this procedure, the usual actuarial fairness principle is not respected because the total premiums collected on a mixed life portfolio (i.e., a portfolio of life insurance products written on both males and females) are not equal to the total premiums charged to the same portfolio before the Gender Directive. The lack of actuarial fairness reflects in difficult reserving procedures and, more in general, in lack of competitiveness.

This paper aims to address the relevant issue of how to price life insurance products by satisfying both actuarial fairness principles and the Gender Directive. We do this in two steps. First, we recall the notion of unisex fairness principle, that should be used to price unisex tariffs for mixed portfolios; at the same time, we define the corresponding unisex fair premium, that respects the unisex fairness principle. Second, we push this process one step further and suggest what should be the underlying unisex mortality model implicitly used to calculate the unisex fair premium: we start by proposing the unisex mortality intensity as a weighted average of the underlying gender-specific mortality intensities, and then we fix the weights in such a way that the actuarial fairness principle is satisfied. Following a well established stream of actuarial literature on the doubly stochastic setup for the stochastic mortality (see [10], [2], [1], [14], [9]), we model the stochastic mortality intensity (i.e., the stochastic force of mortality) of the single gender as an affine process. In particular, we model it as an Ornstein-Uhlenbeck non-mean reverting affine process that is a natural extension of the Gompertz law for the force of mortality. As a consequence, the unisex mortality intensity is a two-factor continuous-time cohort-based mortality model and has the desirable feature of providing closed-form survival probabilities. Finally, we exploit the closed-form survival functions and calibrate the unisex mortality model in practical situations, considering a variety of cohorts and insurance products. The calibrations show that the weights identifying the unisex mortality intensity depend on many factors: mainly (and 
expectedly), the quote of portfolio relative to each gender, but also the age, the cohort, the type of insurance products and the duration of the policy.

Risk management of any kind requires the knowledge of the underlying risk process, and the asset-liability management of a mixed life portfolio makes no exception. Our unisex mortality model provides a picture of the dynamics of the mixed portfolio that insurers have to price, and could facilitate accurate pricing and reserving, improving the companies' competitiveness.

The remaining of the paper is as follows. Section 2 describes the current practice used to calculate unisex tariffs. Section 3 formulates the concepts of unisex fairness principle and unisex fair premium. Section 4 models the unisex mortality intensity as a two-factor stochastic cohort-based continuous-time intensity process, deriving closed-form expressions

for the unisex survival function. Section 5 introduces the concept of fair unisex mortality intensity and fair weighting parameter. Sections 6, 7 and 8 report the numerical part. In Section 6 (i) we calibrate the gender-based mortality intensity processes for a number of UK cohorts, (ii) we compute the fair weighting coefficient for pure endowment, term insurance, endowment and immediate lifetime annuity, (iii) we compare the fair weighting coefficients with the volume-related weights and make comparison across different products and cohorts. In Section 7 we consider periodic premiums. In Section 8 we set the actuarial fairness equivalence on annual basis and calculate time-varying fair weighting coefficients. Section 9 concludes.

\section{Unisex premium: current practice}

It is rather difficult to report on the current actuarial practice for unisex pricing. The main reason is the fact that, to our knowledge, after the Gender Directive the insurance companies have not made clear and detailed disclosure of their pricing process. According to the Italian Guidelines on the application of the Gender Directive to life insurance products ([4]), regarding the unisex tariffs we read:

...the unisex demographic basis ...can be defined on the basis of 
- prevailing risk or

- weighted risk, assuming a prudent mix of insured of both genders that represent an estimate of the theoretical insured population...

If the insurer takes the view only of the prevailing risk, then the premium of the pure endowment will be based only on the females' mortality, and the premium of the term insurance will be based only on the males' mortality. This pricing procedure is safe and prudent, but gives rise to competitiveness and adverse selection issues. Therefore, we consider it inappropriate.

Insurers seem to incorporate both principles of prevailing risk and weighted risk in pricing unisex tariffs. The unisex tariff is currently based on a simple mix of the gender-based life tables, that takes into account the risk covered and gives more weight to the prevailing risk. Therefore, the unisex life table used to price a pure endowment gives more weight to the females' life table, and the unisex life table used to price a term insurance gives more weight to the males' life table. Similar unisex tariffs are adopted in other European countries, too. For instance, in Germany, since the insurance companies have not had long-time experience in determining the weight of female/male in a unisex tariff, the weights are mostly estimated very cautiously. In order to alleviate the estimation errors, unisex tariffs are set in a safe and prudent pricing procedure. Cautious procedures for the unisex tariff are adopted in Spain, too.

It is easy to show that this mixing procedure fails to respect actuarial fairness at the portfolio level. Indeed, using this mixing procedure the total unisex premiums collected on the mixed portfolio are not equal to the total fair premiums that were collected on the two gender-specific subportfolios before the Gender Directive, when the respect of actuarial fairness was not an issue. In this paper, we take the view of the weighted risk, but aim primarily to achieve the actuarial fairness at the portfolio level. 


\section{Unisex fairness principle and unisex fair premium}

In this section we show how the unisex pricing for a mixed portfolio should be done in order to reach actuarial fairness at the global portfolio level.

Consider a life insurance company operating on a time horizon $[0, T], T<\infty$. The company manages a portfolio of identical life insurance policies (e.g., pure endowment with duration $T$, term insurance with duration $T$, etc.) issued to two groups of policyholders: one group with $m \geq 1$ homogenous male policyholders and one group with $n \geq 1$ homogenous female policyholders. All the policyholders are aged $x$ at time 0 . We will call such a portfolio a "mixed portfolio", because it contains both male and female policyholders. For simplicity, let us assume that the cohort mortality tables of the males and females of generation $x$ are given, respectively, by the vectors

$$
\left[p_{x}^{m}, p_{x+1}^{m}, \ldots, p_{\omega-1}^{m}\right]
$$

and

$$
\left[p_{x}^{f}, p_{x+1}^{f}, \ldots, p_{\omega-1}^{f}\right]
$$

where $p_{y}^{i}$ is the one-year survival probability of the individual of gender $i=m$, $f$ at age $y \in\{x, x+1, \ldots, \omega-1\}$, and $\omega$ is the maximal age (e.g. $\omega=120)$. Assume that the fair premium for the male of the policy under consideration, using vector (1), is $P_{m}$, and the fair premium for the female of the policy under consideration, using vector $(2)$, is $P_{f}$.

Disregarding safety loadings and commissions, the total fair premiums collected before December 2012 for the mixed portfolio under consideration was

$$
m \cdot P_{m}+n \cdot P_{f}
$$

because before the Gender Directive the mixed portfolio consisted in two subportfolios, one with $m$ males and fair premium $P_{m}$, and the other with $n$ females and fair premium $P_{f}$. Since December 2012, the most natural way to reach the actuarial fairness at the portfolio level is to charge the same total premiums. Therefore, the unisex premium $P_{u}$ charged by 
the insurance company to each of the $m+n$ policyholders of the mixed portfolio must satisfy

$$
(m+n) \cdot P_{u}=m \cdot P_{m}+n \cdot P_{f}
$$

Therefore, we can define the following unisex fairness principle:

Definition 3.1 (Unisex fairness principle and unisex fair premium). For a given portfolio of $m$ male policyholders and $n$ female policyholders, whose fair premiums are $P_{m}$ and $P_{f}$ respectively, we say that the unisex tariff $P_{u}$ is calculated according to the unisex fairness principle if

$$
P_{u}=w \cdot P_{m}+(1-w) \cdot P_{f}
$$

where

$$
w=\frac{m}{m+n}
$$

is the males' proportion in the portfolio. In this case, the unisex tariff $P_{u}$ is called unisex fair premium.

From this definition, it is evident that the actuarial fairness at portfolio level can be reached only by charging the unisex fair premium.

Remark 1. It is worth noting that the unisex fairness principle and the unisex fair premium just presented, are not novel concepts in actuarial literature. The unisex fairness principle is an application of the more general actuarial equivalence principle at the portfolio level, and the related unisex fair premium is just the premium that reaches the technical equilibrium in a portfolio of non-homogeneous risks. We now give a brief overview of the general framework and refer the interested reader to [11] (Chapters 2 and 9), where an accurate treatment of risk classification and management of portfolios of heterogeneous risks is provided.

Insurance contracts that, according to the insurer, show similar riskiness (in terms of probability of producing a claim) are grouped into the same risk class. Insurance contracts to which the insurer assigns the same premium rate are grouped into the same rating class. Since rating classes are usually fewer than risk classes, the insurer typically needs to select the same premium for two or more risk classes. To this end, the insurer usually applies the 
actuarial equivalence principle among all the policies considered, that belong to different risk classes. Therefore, in the presence of several contracts, the actuarial equivalence principle at the portfolio level reads as

$$
\mathrm{EPV}(\text { total liabilities })=\mathrm{EPV}(\text { total premiums })
$$

where EPV stands for expected present value, "total liabilities" indicates the future liabilities arising from all the policies considered, and "total premiums" indicates the future premiums to be received from all the policies considered. In a very simple case, let us assume that the insurer has a single rating class for two risk classes, A and B. If there are $m$ policies belonging to risk class $A$, for which the actuarially fair premium would be $P_{A}$, and $n$ policies belonging to risk class $B$, for which the actuarially fair premium would be $P_{B}$, the actuarial equivalence principle (5) at the portfolio level for the $m+n$ non-homogeneous policies can be satisfied only if the premium $\bar{P}$ charged to each policy is

$$
\bar{P}=\frac{m P_{A}+n P_{B}}{m+n}
$$

(see [11]). In other words, the technical equilibrium at portfolio level is achieved if and only

if the insurer charges $\bar{P}$ to all policies. It is clear that the unisex fairness principle is a special case of (5), and the unisex fair premium is a special case of (6), in the presence of two subportfolios made by policies belonging to two different risk classes identified by the risk factor gender.

It is interesting and useful to interpret the unisex fair premium $P_{u}$ as the premium of a representative unisex policyholder of the mixed portfolio, and to analyze the relationship between the survival probabilities of the unisex policyholder and those of males and females. The relationship depends on the type of policy and on its duration. As an illustration, we consider four classical life insurance products: (1) pure endowment; (2) term insurance; (3) endowment insurance; (4) immediate lifetime annuity.

\section{Pure endowment}

For the male, the gender-based fair premium of a pure endowment insurance contract which 
pays out a unitary payment if the insured survives the maturity date $T$ is

$$
P_{m}=B(0, T) \cdot{ }_{T} p_{x}^{m}
$$

while that for the female is

$$
P_{f}=B(0, T) \cdot T p_{x}^{f}
$$

where $B(0, T)$ is the financial discount factor from $T$ to 0 and ${ }_{T} p_{x}^{i}, i=m, f$, is the $T$-years survival probability for individual of gender $i$ aged $x$ at time 0 . The unisex fair premium for the pure endowment is

$$
P_{u}=w \cdot P_{m}+(1-w) \cdot P_{f}=B(0, T) \cdot T p_{x}^{u}
$$

where

$$
{ }_{T} p_{x}^{u}=w \cdot \cdot_{T} p_{x}^{m}+(1-w) \cdot{ }_{T} p_{x}^{f}
$$

Therefore, managing a mixed portfolio of pure endowments of duration $T$ written on $m$ males and $n$ females is equivalent to managing a portfolio of $m+n$ pure endowments issued to unisex policyholders whose unisex $T$-years survival probability ${ }_{T} p_{x}^{u}$, given by $(7)$, is a weighted average of the $T$-years survival probabilities of males and females of the portfolio, with weights $w$ and $(1-w)$, that are volume-related and given by (4).

It is easy to show that this pricing procedure does not correspond to mixing the life tables of males and females with volume-related weights. Indeed, simply mixing the life tables with volume-related weights produces (1-year) survival rates that are mixed with volume-related weights, but, for $T \geq 2$ produces $T$-years survival probabilities that are not weighted averages of the gender-based $T$-years survival probabilities with volume-related weights. ${ }^{1}$

\section{Term insurance}

For the male, the gender-based fair premium of a term insurance contract which pays out a unitary death benefit at the end of year of death if the insured does not survive the maturity

\footnotetext{
${ }^{1}$ This is due to the fact that the $T$-years survival probability for age $x$ is the product of the 1-year survival rates at ages $x, x+1, \ldots x+T-1:_{T} p_{x}=p_{x} \cdot p_{x+1} \cdot \ldots \cdot p_{x+T-1}$.
} 
date $T$ is

$$
P_{m}=\sum_{k=0}^{T-1} B(0, k+1) \cdot{ }_{k} / 1 q_{x}^{m}
$$

while that for the female is

$$
P_{f}=\sum_{k=0}^{T-1} B(0, k+1) \cdot{ }_{k / 1} q_{x}^{f}
$$

where ${ }_{k / 1} q_{x}^{i}, i=m, f$, is the $k$-year deferred death probability, i.e. the probability of dying in year $(k, k+1]$ for individual of gender $i$ aged $x$ at time 0 . The unisex fair premium for the term insurance is

$$
P_{u}=w \cdot P_{m}+(1-w) \cdot P_{f}=\sum_{k=0}^{T-1} B(0, k+1) \cdot{ }_{k / 1} q_{x}^{u}
$$

where

$$
{ }_{k / 1} q_{x}^{u}=w \cdot_{k / 1} q_{x}^{m}+(1-w){ }_{k / 1} q_{x}^{f} \quad \text { for all } k=0,1, \ldots, T-1 .
$$

Therefore, managing a portfolio of term insurances of duration $T$ written on $m$ males and $n$ females is equivalent to managing a portfolio of $m+n$ term insurances issued to unisex policyholders whose unisex $k$-year deferred death probability ${ }_{k / 1} q_{x}^{u}$, given by (8), is a weighted average of the $k$-year deferred death probabilities of males and females of the portfolio, for every $k=0,1, \ldots, T-1$, with weights $w$ and $(1-w)$, that are volume-related and given by (4).

As before, it is easy to show that this pricing procedure does not correspond to mixing the life tables of males and females with volume-related weights.

\section{Endowment insurance}

Combining the above two contracts, we obtain an endowment insurance contract which pays out a unitary death benefit at the end of year of death if the insured does not survive the maturity date $T$ or a unitary survival benefit if the insured survives $T$. For the male, the 
gender-based fair premium of this contract is

$$
P_{m}=\sum_{k=0}^{T-1} B(0, k+1) \cdot_{k / 1} q_{x}^{m}+{ }_{T} p_{x}^{m} \cdot B(0, T)
$$

while that for the female is

$$
P_{f}=\sum_{k=0}^{T-1} B(0, k+1) \cdot{ }_{k / 1} q_{x}^{f}+{ }_{T} p_{x}^{f} \cdot B(0, T) .
$$

The unisex fair premium for the endowment insurance is

$$
P_{u}=w \cdot P_{m}+(1-w) \cdot P_{f}=\sum_{k=0}^{T-1} B(0, k+1) \cdot{ }_{k / 1} q_{x}^{u}+{ }_{T} p_{x}^{u} \cdot B(0, T)
$$

where

$$
{ }_{k / 1} q_{x}^{u}=w \cdot{ }_{k / 1} q_{x}^{m}+(1-w) \cdot_{k / 1} q_{x}^{f} \quad \text { for all } k=0,1, \ldots, T-1,
$$

and

$$
{ }_{T} p_{x}^{u}=w \cdot{ }_{T} p_{x}^{m}+(1-w) \cdot{ }_{T} p_{x}^{f}
$$

Therefore, managing a portfolio of endowments of duration $T$ written on $m$ males and $n$ females is equivalent to managing a portfolio of $m+n$ endowments issued to unisex policyholders whose unisex $k$-year deferred death probability ${ }_{k / 1} q_{x}^{u}$ is a weighted average of the $k$-year deferred death probabilities of males and females of the portfolio, with weights $w$ and $(1-w)$, that are volume-related and given by (4), and whose unisex $T$-years survival probability ${ }_{T} p_{x}^{u}$ is a weighted average of the $T$-years survival probabilities of males and females of the portfolio, with weights $w$ and $(1-w)$.

\section{Immediate lifetime annuity}

For the male, the gender-based fair premium of an immediate lifetime annuity with unitary 
benefit (paid in advance) is

$$
P_{m}=\sum_{k=0}^{\omega-x-1} B(0, k) \cdot{ }_{k} p_{x}^{m}
$$

while that for the female is

$$
P_{f}=\sum_{k=0}^{\omega-x-1} B(0, k) \cdot{ }_{k} p_{x}^{f},
$$

where $\omega$ is the maximal age. The unisex fair premium for the immediate lifetime annuity is

$$
P_{u}=w \cdot P_{m}+(1-w) \cdot P_{f}=\sum_{k=0}^{\omega-x-1} B(0, k) \cdot_{k} p_{x}^{u}
$$

where

$$
{ }_{k} p_{x}^{u}=w{ }_{k} p_{x}^{m}+(1-w){ }_{k} p_{x}^{f} \quad \text { for all } k=0,1, \ldots, \omega-x-1 .
$$

Therefore, managing a portfolio of lifetime annuities issued to $m$ males and $n$ females is equivalent to managing a portfolio of $m+n$ annuities issued to unisex individuals whose unisex $k$-years survival probability is a weighted average of the $k$-years survival probabilities of males and females of the portfolio, for every $k=0,1, \ldots, \omega-x-1$, with weights $w$ and $(1-w)$, that are volume-related and given by (4).

\section{Unisex stochastic mortality model}

In the previous section, we have seen that the achievement of actuarial fairness boils down to a simple volume-related mixing of survival probabilities or deferred death probabilities, depending on the nature of the underlying contract. It is unclear whether this corresponds precisely to a volume-related mixing of the two underlying gender-specific mortality models. Generally, this is not the case. In this section, we propose a two-factor stochastic continuoustime unisex mortality model that is a weighted average of the two underlying gender-specific stochastic mortality models. In Section 5 , we will show how to select the weights in order to have a unisex mortality model that produces exactly the unisex fair premium calculated according to the unisex fairness principle of Definition 3.1. 
Let us introduce a complete filtered probability space $(\Omega, \mathfrak{F}, \mathbb{P})$ and a filtration $\mathcal{F}_{t}$ of sub- $\sigma$ algebras representing the state of information at time $0 \leq t \leq T$. As in Section 2 , consider an insurance company that manages a mixed portfolio with $m$ male policyholders and $n$ females policyholders, who have the same age $x$ at time 0 . For notational convenience, and because we are assuming that males and females of the mixed portfolio belong to the same cohort and have the same initial age $x$, throughout this section and Section 5 we will omit the dependence on $x$ of the mortality processes. The stochastic mortality intensities of the two groups are described by two different Ornstein-Uhlenbeck processes (OU processes) with positive drift and no mean reversion: ${ }^{2}$

$$
\begin{aligned}
d \lambda^{m}(t) & =\mu_{m} \lambda^{m}(t) d t+\sigma_{m} d W^{m}(t), \\
d \lambda^{f}(t) & =\mu_{f} \lambda^{f}(t) d t+\sigma_{f} d W^{f}(t),
\end{aligned}
$$

where $\mu_{i}>0$ and $\sigma_{i}>0$ for $i=m, f$, and $W_{m}$ and $W_{f}$ are two standard Brownian motions under the real world measure $\mathbb{P}$, correlated with a correlation coefficient $\rho$. So, there is a Brownian motion $W^{\perp m}$, independent of $W^{m}$ such that $W^{f}=\rho W^{m}+\sqrt{1-\rho^{2}} W^{\perp m}$. The OU process for the mortality intensity is a natural stochastic generalization of the Gompertz law for the force of mortality and was introduced by [9], where its properties and the conditions for its biological reasonableness have been discussed.

Standard properties of affine processes allow us to write the survival probability of a male

\footnotetext{
${ }^{2}$ To keep the treatment as simple as possible, in this paper we do not present all the mathematical arsenal that is behind the introduction of affine processes for the stochastic mortality intensity, i.e. the doubly stochastic setup for the stochastic mortality. To be precise, in this framework the time of death is modelled as the first jump time of a doubly stochastic processes with intensity $\lambda$. A complete exposition is beyond the scope of this paper, and we refer the interested reader to the papers on the doubly stochastic setup cited in Section 1.
} 
and a female policyholder in closed-form (see [9]):

$$
\begin{aligned}
S^{i}(t, T) & =E\left[\exp \left\{-\int_{t}^{T} \lambda^{i}(u) d u\right\} \mid \mathcal{F}_{t}\right]=\exp \left\{\alpha_{i}(T-t)+\beta_{i}(T-t) \lambda^{i}(t)\right\}, i=m, f \\
\alpha_{i}(t) & =\frac{\sigma_{i}^{2}}{2 \mu_{i}^{2}} t-\frac{\sigma_{i}^{2}}{\mu_{i}^{3}} e^{\mu_{i} t}+\frac{\sigma_{i}^{2}}{4 \mu_{i}^{3}} e^{2 \mu_{i} t}+\frac{3 \sigma_{i}^{2}}{4 \mu_{i}^{3}}, i=m, f \\
\beta_{i}(t) & =\frac{1}{\mu_{i}}\left(1-e^{\mu_{i} t}\right), i=m, f .
\end{aligned}
$$

The key idea of this paper is to model the mortality intensity of a representative unisex policyholder of the mixed portfolio as a weighted average of the males' and females' mortality intensities, according to the following definition:

Definition 4.1 (Unisex mortality intensity). For a mixed portfolio of male and female policyholders, whose stochastic mortality intensities are $\lambda^{m}$ and $\lambda^{f}$ respectively, we define the $\xi$-driven unisex mortality intensity by mixing the male and female intensities with the weight $\xi \in[0,1]:$

$$
\lambda_{\xi}^{u}(t)=\xi \lambda^{m}(t)+(1-\xi) \lambda^{f}(t)
$$

Clearly, Definition 4.1 defines a family of unisex mortality intensities, the members of which are identified by the weight $\xi \in[0,1]$. The appropriate identification of the weight $\xi$ is the subject of the next section.

From (9) and (11), we obtain

$$
d \lambda_{\xi}^{u}(t)=\left(\xi \mu_{m} \lambda^{m}(t)+(1-\xi) \mu_{f} \lambda^{f}(t)\right) d t+\xi \sigma_{m} d W^{m}(t)+(1-\xi) \sigma_{f} d W^{f}(t)
$$

Note that $\lambda_{\xi}^{u}$ does not follow a simple OU process like $\lambda^{m}$ and $\lambda^{f}$, because in general $\mu_{m}$ is different from $\mu_{f}$. Despite this, the survival probability related to the mixed mortality intensity $\lambda_{\xi}^{u}$ can still be computed nicely. ${ }^{3}$

Proposition 4.2. Conditional on $t$, the survival probability for the remaining time $\tau=T-t$

\footnotetext{
${ }^{3}$ In the following, in order to simplify notation, we will omit the subscript $\xi$ in the functions $\alpha, \beta_{1}$ and $\beta_{2}$.
} 
related to the mixed mortality intensity $\lambda_{\xi}^{u}$ in (11) is given by

$$
S_{\xi}^{u}(t, T)=E\left[\exp \left\{-\int_{t}^{T} \lambda_{\xi}^{u}(s) d s \mid \mathcal{F}_{t}\right\}\right]=\exp \left\{\alpha_{u}(\tau)+\beta_{1, u}(\tau) \lambda^{m}(t)+\beta_{2, u}(\tau) \lambda^{f}(t)\right\}
$$

with

$$
\begin{gathered}
\beta_{1, u}(\tau)=\frac{\xi}{\mu_{m}}\left(1-e^{\mu_{m} \tau}\right), \\
\beta_{2, u}(\tau)=\frac{1-\xi}{\mu_{f}}\left(1-e^{\mu_{f} \tau}\right),
\end{gathered}
$$

and

$$
\begin{aligned}
& \alpha_{u}(\tau)=\frac{\sigma_{m}^{2} \xi^{2}}{4 \mu_{m}^{3}}\left[\left(e^{\mu_{m} \tau}-2\right)^{2}+2 \mu_{m} \tau-1\right]+\frac{\sigma_{f}^{2}(\xi-1)^{2}}{4 \mu_{f}^{3}}\left[\left(e^{\mu_{f} \tau}-2\right)^{2}+2 \mu_{f} \tau-1\right] \\
& -\frac{\rho \sigma_{m} \sigma_{f} \xi(\xi-1)}{\mu_{m}^{2} \mu_{f}^{2}\left(\mu_{m}+\mu_{f}\right)}\left\{\mu_{m}^{2}\left(1-e^{\mu_{f} \tau}\right)+\mu_{f}^{2}\left(1-e^{\mu_{m} \tau}\right)+\mu_{m} \mu_{f}\left[\left(1-e^{\mu_{m} \tau}\right)\left(1-e^{\mu_{f} \tau}\right)+\left(\mu_{m}+\mu_{f}\right) \tau\right]\right\} .
\end{aligned}
$$

Proof. Following [3], and the fact that $\lambda^{u}$ is a linear function of the two state variables $\lambda^{m}$ and $\lambda^{f}$, the survival probability related to the mixed mortality intensity $\lambda^{u}$ can be expressed as follows:

$$
E\left[\exp \left\{-\int_{t}^{T} \lambda_{\xi}^{u}(s) d s\right\} \mid \mathcal{F}_{t}\right]=\exp \left\{\alpha_{u}(t)+\beta_{1, u}(t) \lambda^{m}(t)+\beta_{2, u}(t) \lambda^{f}(t)\right\},
$$

where $\alpha_{u}(t), \beta_{1, u}(t)$ and $\beta_{2, u}(t)$ follow some ODEs. In order to find out the ODEs, we need to discuss the following conditions.

- The mean of the state variables shall satisfy the affine structure:

$$
\left(\begin{array}{c}
\mu_{m} \lambda^{m} \\
\mu_{f} \lambda^{f}
\end{array}\right):=K_{0}+K_{1}\left(\begin{array}{c}
\lambda^{m} \\
\lambda^{f}
\end{array}\right) \Rightarrow K_{0}=0, K_{1}=\left(\begin{array}{cc}
\mu_{m} & 0 \\
0 & \mu_{f}
\end{array}\right)
$$


- The volatility of the state variables shall satisfy the affine structure:

$$
\begin{aligned}
& \left(\begin{array}{cc}
\sigma_{m} & 0 \\
\rho \sigma_{f} & \sqrt{1-\rho^{2}} \sigma_{f}
\end{array}\right)\left(\begin{array}{cc}
\sigma_{m} & \rho \sigma_{f} \\
0 & \sqrt{1-\rho^{2}} \sigma_{f}
\end{array}\right)=\left(\begin{array}{cc}
\sigma_{m}^{2} & \rho \sigma_{f} \sigma_{m} \\
\rho \sigma_{f} \sigma_{m} & \sigma_{f}^{2}
\end{array}\right) \\
:= & H_{0}+H_{1}\left(\begin{array}{c}
\lambda^{m} \\
\lambda^{f}
\end{array}\right) \Rightarrow H_{0}=\left(\begin{array}{cc}
\sigma_{m}^{2} & \rho \sigma_{f} \sigma_{m} \\
\rho \sigma_{f} \sigma_{m} & \sigma_{f}^{2}
\end{array}\right), H_{1}=0 .
\end{aligned}
$$

- $\lambda^{u}$ is a linear function of $\lambda^{m}$ and $\lambda^{f}$ :

$$
\left(\begin{array}{ll}
\xi & 1-\xi
\end{array}\right)\left(\begin{array}{c}
\lambda^{m} \\
\lambda^{f}
\end{array}\right):=\rho_{0}+\rho_{1}\left(\begin{array}{c}
\lambda^{m} \\
\lambda^{f}
\end{array}\right) \Rightarrow \rho_{0}=0, \rho_{1}=\left(\begin{array}{ll}
\xi & 1-\xi
\end{array}\right)
$$

After having determined all the necessary parameters, we can write down the conditions for $\alpha_{u}(t), \beta_{1, u}(t)$ and $\beta_{2, u}(t)$ :

$$
\begin{aligned}
\beta_{1, u}^{\prime}(t) & =\xi-\mu_{m} \beta_{1, u}(t), \text { with } \beta_{1, u}(T)=0 \\
\beta_{2, u}^{\prime}(t) & =(1-\xi)-\mu_{f} \beta_{2, u}(t), \text { with } \beta_{2, u}(T)=0 \\
\alpha_{u}^{\prime}(t) & =-\frac{1}{2}\left(\beta_{1, u}(t) \beta_{2, u}(t)\right) H_{0}\left(\begin{array}{c}
\beta_{1, u}(t) \\
\beta_{2, u}(t)
\end{array}\right) \\
& =-\frac{1}{2}\left(\beta_{1, u}^{2}(t) \sigma_{m}^{2}+\beta_{2, u}^{2}(t) \sigma_{f}^{2}+2 \beta_{1, u} \beta_{2, u} \rho \sigma_{f} \sigma_{m}\right), \text { with } \alpha_{u}(T)=0 .
\end{aligned}
$$

When we consider survival probabilities, we do not have terminal conditions, $\alpha_{u}(T)=$ $\beta_{1, u}(T)=\beta_{2, u}(T)=0$. Instead it holds $\alpha_{u}(0)=\beta_{1, u}(0)=\beta_{2, u}(0)=0$. Noticing the fact that $\beta_{i}^{\prime}(\tau)=-\beta_{i}^{\prime}(t), \quad i=1,2$, and $\alpha_{u}^{\prime}(\tau)=-\alpha_{u}^{\prime}(t)$, the above three ODEs can be transformed to

$$
\begin{aligned}
& \beta_{1, u}^{\prime}(\tau)=\mu_{m} \beta_{1, u}(\tau)-\xi, \text { with } \beta_{1, u}(0)=0 \\
& \beta_{2, u}^{\prime}(\tau)=\mu_{f} \beta_{2, u}(\tau)-(1-\xi), \text { with } \beta_{2, u}(0)=0 \\
& \alpha_{u}^{\prime}(\tau)==\frac{1}{2}\left(\beta_{1, u}^{2}(\tau) \sigma_{m}^{2}+\beta_{2, u}^{2}(\tau) \sigma_{f}^{2}+2 \beta_{1, u} \beta_{2, u} \rho \sigma_{f} \sigma_{m}\right), \text { with } \alpha_{u}(0)=0 .
\end{aligned}
$$

with $\tau:=T-t$. Solving the ODEs leads to the expressions for $\beta_{1, u}(\tau), \beta_{2, u}(\tau)$ and $\alpha_{u}(\tau)$. 
Figure 1 illustrates a trajectory for the intensity of mortality and Figure 2 plots the survival functions, for males and females, and the joint one for UK population, cohort 1950, initial age 45 , with $\xi=0.5$ and $\rho=0.95$. We have used the maximal age 120 . As noticed, the male and female force of mortality follows an OU process which is an exponential with a random noise, and the joint force of mortality is just the weighted sum of the male and female forces of mortality. Like using Gompertz-Makeham force of mortality, the survival probabilities approach zero for high ages.

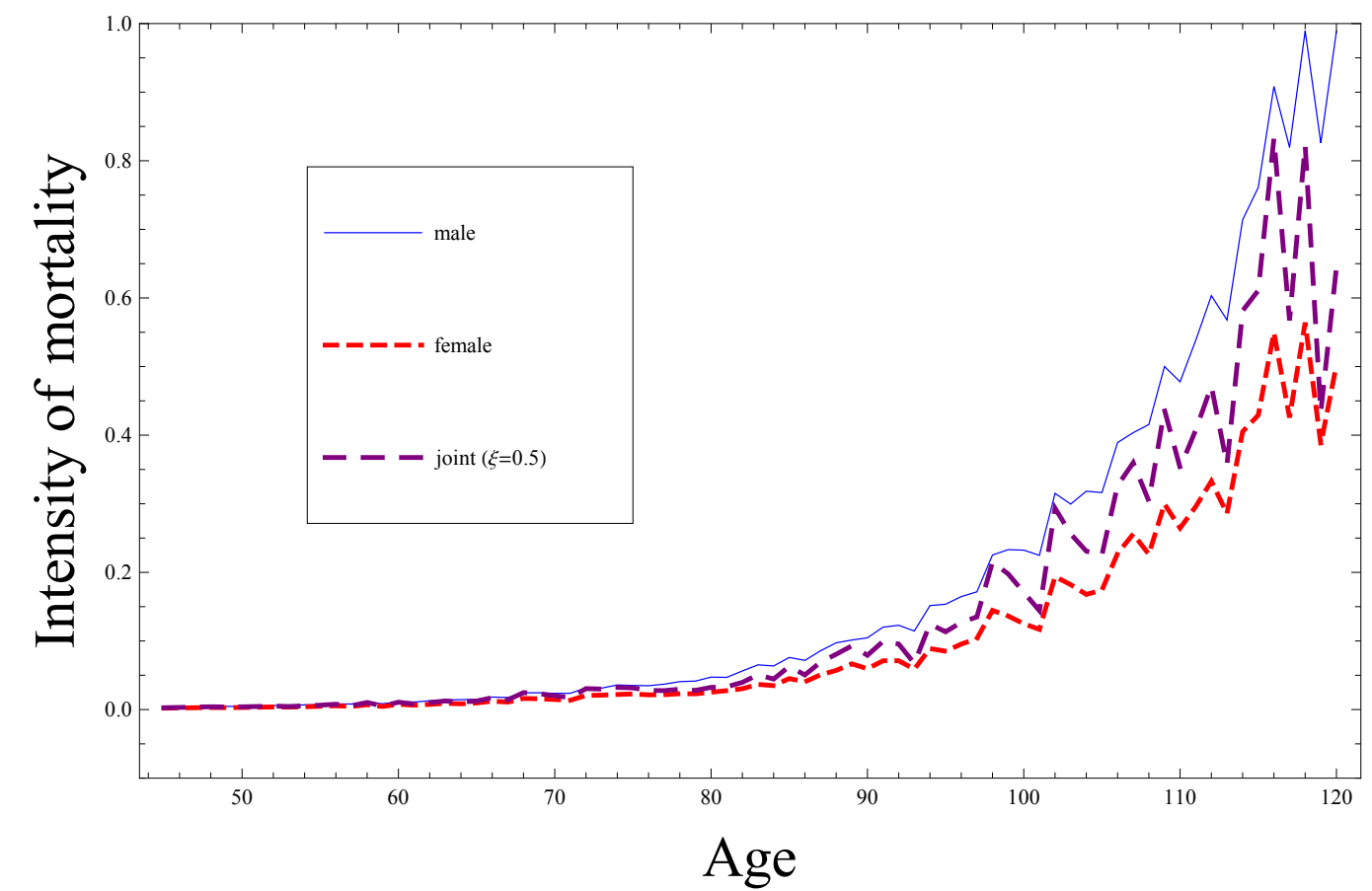

Figure 1: A trajectory for the force of mortality using UK population, cohort 1940, initial age 45 (see values of Table 1) (source: HMD-2013).

Remark 2. As a special case, if we set $\sigma_{m}=0$ and $\sigma_{f}=0$ in the stochastic framework, we retrieve the deterministic mortality model where the males' and the females' forces of mortality are Gompertz:

$$
\lambda_{i}(t)=\lambda_{i}(0) e^{\mu_{i} t}, i=m, f
$$




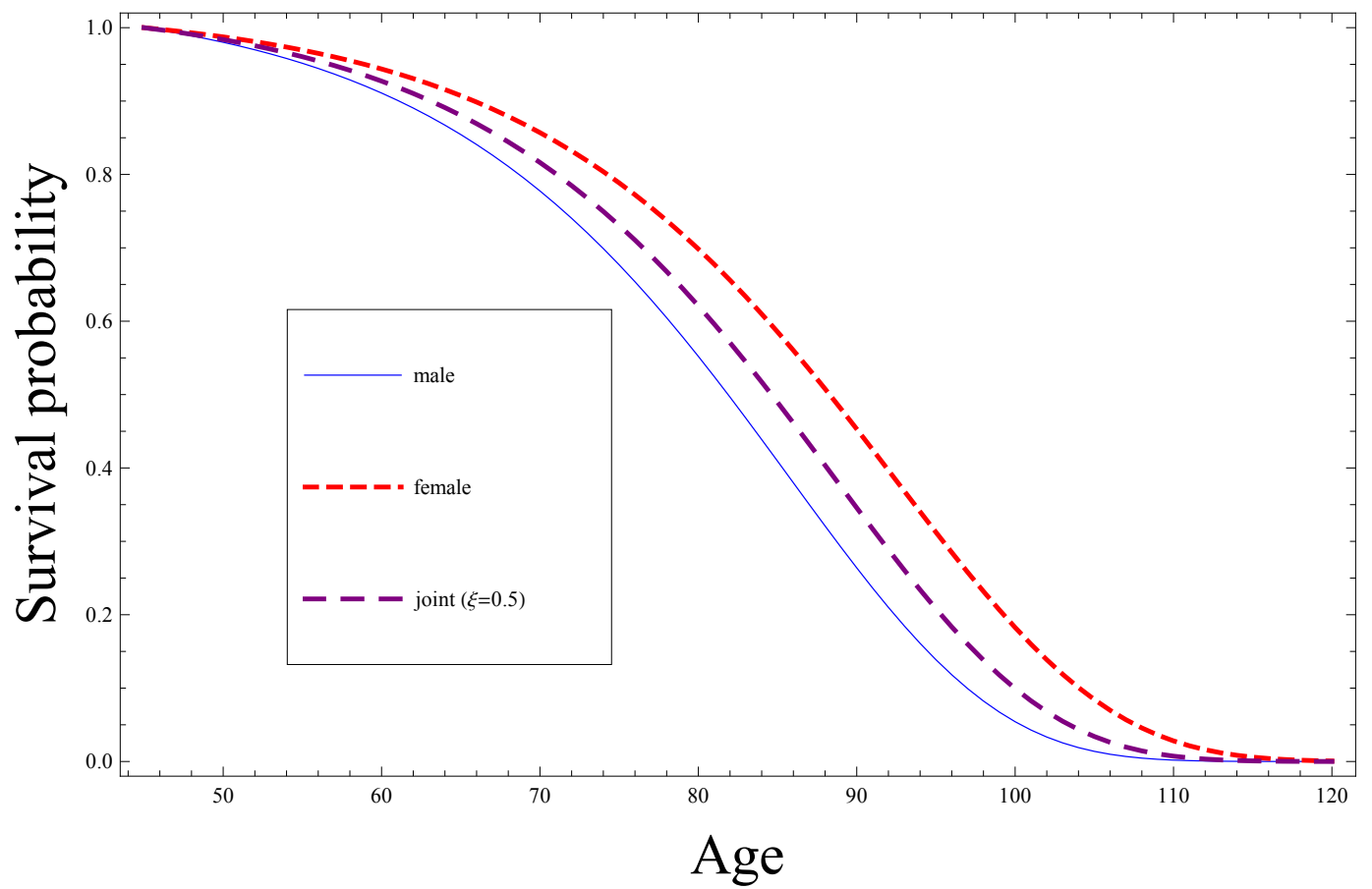

Figure 2: Survival probabilities relative to UK population, cohort 1940, initial age 45 (see values of Table 1) (source: HMD-2013).

Accordingly, the deterministic survival probabilities are:

$$
\begin{aligned}
S^{i}(t, T) & =\exp \left\{\frac{\lambda_{i}(0)}{\mu_{i}}\left(e^{\mu_{i} t}-e^{\mu_{i} T}\right)\right\}, i=m, f \\
S_{\xi}^{u}(t, T) & =\exp \left\{\frac{\xi \lambda_{m}(0)}{\mu_{m}}\left(e^{\mu_{m} t}-e^{\mu_{m} T}\right)+\frac{(1-\xi) \lambda_{f}(0)}{\mu_{f}}\left(e^{\mu_{f} t}-e^{\mu_{f} T}\right)\right\} .
\end{aligned}
$$

\section{$5 \quad$ Fair unisex mortality intensity}

In this section, we assume that the mixed life insurance portfolio is given and we aim to determine the fair unisex mortality intensity that is consistent with the unisex fairness principle (3) for that portfolio.

Regarding notation, we observe that the fair premium of a life insurance policy is just a function $\operatorname{Prem}(\cdot)$ of the underlying mortality intensity process. Therefore, assuming that 
the mortality intensities of male, females and unisex policyholders are given by $\lambda^{m}, \lambda^{f}$ and $\lambda_{\xi}^{u}$ respectively, we shall naturally call

$$
P_{m}:=\operatorname{Prem}\left(\lambda^{m}\right) \quad P_{f}:=\operatorname{Prem}\left(\lambda^{f}\right) \quad P_{u}:=\operatorname{Prem}\left(\lambda_{\xi}^{u}\right)
$$

With this new notation, we can define what is the fair unisex mortality intensity that produces the unisex fair premium:

Definition 5.1 (Fair unisex mortality intensity). For a given portfolio of $m$ male policyholders and $n$ female policyholders, whose fair gender-based premiums are Prem $\left(\lambda^{m}\right)$ and $\operatorname{Prem}\left(\lambda^{f}\right)$ respectively, we say that $\lambda_{\xi^{*}}^{u}$ is a fair unisex mortality intensity if the corresponding unisex premium

$$
\operatorname{Prem}\left(\lambda_{\xi^{*}}^{u}\right)=\operatorname{Prem}\left(\xi^{*} \lambda^{m}+\left(1-\xi^{*}\right) \lambda^{f}\right)
$$

is fair, i.e. it satisfies the unisex fairness principle (3):

$$
m \cdot \operatorname{Prem}\left(\lambda^{m}\right)+n \cdot \operatorname{Prem}\left(\lambda^{f}\right)=(m+n) \cdot \operatorname{Prem}\left(\xi^{*} \lambda^{m}+\left(1-\xi^{*}\right) \lambda^{f}\right) .
$$

The weight $\xi^{*}$ is said to be fair mortality intensity mixing parameter.

In other words, among the unisex mortality intensities of the family of Definition 4.1, the fair unisex mortality intensity computed with $\xi^{*}$ respects actuarial fairness.

Due to the closed-form expressions for the survival functions $S^{m}(\cdot, \cdot), S^{f}(\cdot, \cdot)$ and $S_{\xi}^{u}(\cdot, \cdot)$, the numerical computation of the fair $\xi^{*}$ is not complicated, once the mortality intensity processes $\lambda^{m}$ and $\lambda^{f}$ have been calibrated. But we need to point out that despite its closedform, the survival probably is a rather complex function of the mortality intensity mixing parameter $\xi$, thus the fair $\xi^{*}$ has to be determined with numerical procedures, and therefore the practical implementation of the model can be limited.

In the following, as an illustrative example, we will again consider the four insurance products: pure endowment, term insurance, endowment insurance and immediate lifetime annuity. We will assume that the discount factor is $B(0, T)=e^{-r T}$, where $r \geq 0$ is the risk free interest rate. 


\section{Pure endowment}

Consider the case in which $m$ males and $n$ females with the same age buy a pure endowment insurance contract which pays out a unitary payment if they survive the maturity date $T$. According to (15), the fair $\xi^{*}$ for the mixed portfolio with $m+n$ policyholders results by setting:

$$
m \cdot e^{-r T} \cdot S^{m}(0, T)+n \cdot e^{-r T} \cdot S^{f}(0, T)=(m+n) \cdot e^{-r T} \cdot S_{\xi^{*}}^{u}(0, T)
$$

where the survival functions $S^{m}(0, T)$ and $S^{f}(0, T)$ are given by (10) (with $i=m$, $f$ respectively), and the unisex survival function $S_{\xi}^{u}(0, T)$ is given by (13). The above equation can be reduced to

$$
m \cdot S^{m}(0, T)+n \cdot S^{f}(0, T)=(m+n) \cdot S_{\xi^{*}}^{u}(0, T),
$$

from which $\xi^{*}$ can be computed numerically. Notice that in this case the fair $\xi^{*}$ does not depend on the risk free rate $r$.

\section{Term insurance}

Consider the case in which $m$ males and $n$ females with the same age buy a term insurance contract which pays out a unitary death benefit at the end of year of death if the insureds do not survive the maturity date $T$. The fair $\xi^{*}$ for the mixed portfolio with $m+n$ policyholders results by setting:

$$
\begin{aligned}
& m \cdot \sum_{i=0}^{T-1} e^{-r(i+1)}\left(S^{m}(0, i)-S^{m}(0, i+1)\right)+n \cdot \sum_{i=0}^{T-1} e^{-r(i+1)}\left(S^{f}(0, i)-S^{f}(0, i+1)\right) \\
& =(m+n) \cdot \sum_{i=0}^{T-1} e^{-r(i+1)}\left(S_{\xi^{*}}^{u}(0, i)-S_{\xi^{*}}^{u}(0, i+1)\right) .
\end{aligned}
$$

Notice that in this case the fair $\xi^{*}$ depends on the risk free rate $r$.

\section{Endowment insurance}


Consider the case in which $m$ males and $n$ females with the same age buy an endowment insurance contract with a maturity date $T$ which pays out a unitary benefit upon death/survival. The fair $\xi^{*}$ for the mixed portfolio with $m+n$ policyholders results by setting:

$$
\begin{aligned}
& m \cdot \sum_{i=0}^{T-1} e^{-r(i+1)}\left(S^{m}(0, i)-S^{m}(0, i+1)\right)+m \cdot e^{-r T} S^{m}(0, T) \\
& +n \cdot \sum_{i=0}^{T-1} e^{-r(i+1)}\left(S^{f}(0, i)-S^{f}(0, i+1)\right)+n \cdot e^{-r T} S^{f}(0, T) \\
& =(m+n) \cdot \sum_{i=0}^{T-1} e^{-r(i+1)}\left(S_{\xi^{*}}^{u}(0, i)-S_{\xi^{*}}^{u}(0, i+1)\right)+(m+n) \cdot e^{-r T} S_{\xi^{*}}^{u}(0, T) .
\end{aligned}
$$

Naturally, also in this case the fair $\xi^{*}$ depends on the risk free rate $r$.

\section{Immediate lifetime annuity}

Finally, let us come back to the immediate lifetime annuity product, which pays out an annual unitary pension income 1 at the beginning of the year, starting immediately. In this case, the fair $\xi^{*}$ results from

$$
m \sum_{i=0}^{\omega-x-1} e^{-r i} S^{m}(0, i)+n \sum_{i=0}^{\omega-x-1} e^{-r i} S^{f}(0, i)=(m+n) \sum_{i=0}^{\omega-x-1} e^{-r i} S_{\xi^{*}}^{u}(0, i) .
$$

Notice that also in this case the fair $\xi^{*}$ depends on the risk free rate $r$.

Apart from the pure endowment contracts, all the resulting $\xi^{*}$ depend on the interest rate. But it shall be pointed out that this is not a special property resulting from the stochastic modelling of the force of mortality. If we plug in the deterministic survival probabilities as in Remark 2 in the above Equations (17), (18), and (19), we still have the effect of the risk-free rate on the fair mortality intensity mixing parameter $\xi^{*}$. Clearly, also in the deterministic case, $\xi^{*}$ cannot be determined explicitly and has to be found numerically. Finally, unlike the portfolio weight (4), note that the fair mortality intensity parameter $\xi^{*}$ depends on the choice of the product crucially. This will become clear in Section 6 , where $\xi^{*}$ will be calculated 
with a variety of products.

Remark 3. It is important to stress that the stochastic mortality model introduced in Section 4 is only one special case among the mortality models that can support the unisex fairness principle. Clearly, every mortality model can support the unisex fairness principle. Together with the deterministic setup illustrated in Remark 2, another sensible choice could be considering two gender-specific projected mortality tables, say $\left\{p_{y}^{m}\right\}_{y=x, x+1, \ldots, \omega-1}$ and $\left\{p_{y}^{f}\right\}_{y=x, x+1, \ldots, \omega-1}$ for males and females aged $x$, respectively. One can mix the genderspecific survival rates with a mixing coefficient $\phi$ and define the unisex projected survival rates as follows:

$$
p_{y}^{u n i s e x}=\phi p_{y}^{m}+(1-\phi) p_{y}^{f} \quad y=x, x+1, \ldots, \omega-1 .
$$

A fair mortality mixing parameter $\phi^{*}$ will then result from satisfying the unisex fairness principle introduced in Definition 3.1. Clearly, the resulting fair $\phi^{*}$ will differ from the fair $\xi^{*}$. Similarly to $\xi^{*}$, the fair $\phi^{*}$ will vary among different contract products.

The analysis of this section, and in particular Equations (16)-(19), indicates that the computation of $\xi^{*}$ has to be done numerically, and the relationship between the fair $\xi^{*}$ and the portfolio weight $w$ is highly non-linear and convoluted. In general, very little can be said about this relationship, which is indeed the object of the numerical analysis of Section 6 . However, it is possible to state a fairly general result in the case of deterministic forces of mortality, and not necessarily Gompertz-type. In this case, if the unisex force of mortality is defined as in Definition 4.1 and the fair unisex force of mortality is defined as in Definition 5.1 , then, under reasonable assumptions, in the case of the pure endowment we have $\xi^{*} \leq w$. This is proved in the next proposition. ${ }^{4}$

Proposition 5.2. Let $\lambda^{m}(t)$ and $\lambda^{f}(t)$ be the force of mortality of the males and the females, respectively. Let

$$
\lambda_{\xi^{*}}^{u}(t)=\xi^{*} \lambda^{m}(t)+\left(1-\xi^{*}\right) \lambda^{f}(t)
$$

be the fair unisex force of mortality satisfying Definition 5.1 for a portfolio of pure endowment with maturity $T$ years, and let $w \in[0,1]$ be the proportion of males in the portfolio. If

\footnotetext{
${ }^{4}$ We are grateful to Pietro Millossovich for suggesting this result.
} 
$\lambda^{m}(t) \geq \lambda^{f}(t)$ for all $t \in[0, T]$, then

$$
\xi^{*} \leq w
$$

Proof. Let ${ }_{T} p_{x}^{m}$ and ${ }_{T} p_{x}^{f}$ be the $T$-years survival probability for the male and the female, respectively. Then, (16) yields

$$
e^{-\int_{0}^{T}\left[\xi^{*} \lambda^{m}(t)+\left(1-\xi^{*}\right) \lambda^{f}(t)\right] d t}=w_{T} p_{x}^{m}+(1-w)_{T} p_{x}^{f}
$$

Because the weighted arithmetic mean is greater than the weighted geometric mean, we have

$$
w_{T} p_{x}^{m}+(1-w)_{T} p_{x}^{f} \geq\left({ }_{T} p_{x}^{m}\right)^{w}\left({ }_{T} p_{x}^{f}\right)^{1-w},
$$

hence

$$
e^{-\int_{0}^{T}\left[\xi^{*} \lambda^{m}(t)+\left(1-\xi^{*}\right) \lambda^{f}(t)\right] d t} \geq\left({ }_{T} p_{x}^{m}\right)^{w}\left({ }_{T} p_{x}^{f}\right)^{1-w}=e^{-\int_{0}^{T}\left[w \lambda^{m}(t)+(1-w) \lambda^{f}(t)\right] d t} .
$$

Therefore

$$
-\int_{0}^{T}\left[\xi^{*} \lambda^{m}(t)+\left(1-\xi^{*}\right) \lambda^{f}(t)\right] d t \geq-\int_{0}^{T}\left[w \lambda^{m}(t)+(1-w) \lambda^{f}(t)\right] d t
$$

that is equivalent to

$$
\int_{0}^{T}\left(w-\xi^{*}\right)\left[\lambda^{m}(t)-\lambda^{f}(t)\right] d t \geq 0
$$

Finally, since

$$
\lambda^{m}(t)-\lambda^{f}(t) \geq 0 \quad \text { for } t \in[0, T]
$$

we have

$$
w \geq \xi^{*},
$$

as desired.

Proposition 5.2 points out that, in the case of pure endowment, with deterministic forces of mortality the fair mixing coefficient $\xi^{*}$ that is equivalent to the proportion $w$ of males in the portfolio is lower than the proportion $w$ itself, provided that the force of mortality of males is larger than that of females. The extension of this result to a stochastic mortality 
model and to life insurance products other than the pure endowment is not trivial. The numerical analysis of Section 6 will shed more light on the relationship between $\xi^{*}$ and $w$.

\section{$6 \quad$ Numerical analysis}

In this section, we analyze numerically how the fair $\xi^{*}$ depends on the parameters of the model. In Section 6.1 we calibrate the parameters $\mu_{m}, \mu_{f}, \sigma_{m}, \sigma_{f}$ for a number of cohorts and ages. In Section 6.2.1, we first fix the value of $\rho$ and determine, for all cohorts, the fair $\xi^{*}$ for different insurance products and different durations; we analyze how $\xi^{*}$ depends on the insurance product, on the duration and on the cohort; we then let $\rho$ taking values in $[-1,1]$ and, for each cohort, we analyze how $\xi^{*}$ depends on $\rho$ for different insurance products.

\subsection{Calibration procedure}

We have taken cohort death rates from the Human Mortality Database (Last-modified: 06May-2013) (HMD hereafter) for four different cohorts belonging to the UK population:

- cohort born in 1950 with initial age 35;

- cohort born in 1940 with initial age 45;

- cohort born in 1930 with initial age 55;

- cohort born in 1920 with initial age 65.

The calibration procedure is the following. For each cohort of initial age $x$ and each gender $i=m, f$ we have extrapolated from the HMD twenty observed survival probabilities ${ }_{t} \hat{p}_{x}^{i}$, $t=1, \ldots, 20$; then, we have calibrated the values of the parameters $\mu_{m}, \mu_{f}, \sigma_{m}, \sigma_{f}$ that appear in the theoretical survival functions $S_{x}^{m}(0, t)$ and $S_{x}^{f}(0, t)$ given by $(10)$ by minimizing the following mean square error

$$
\frac{1}{20} \sum_{t=1}^{20}\left({ }_{t} \hat{p}_{x}^{i}-S_{x}^{i}(0, t)\right)^{2}
$$


for $i=m, f$. In all cases, the value of the initial observed intensity $\lambda_{x}^{i}(0)$ is set equal to $-\ln \hat{p}_{x}^{i}$. This calibration procedure is standard and in the actuarial context has been used to calibrate the OU intensity process in [7], [8] and [9]. Table 1 reports the calibrated values of the parameters for the generations 1950 and 1940 for both genders and Table 2 those for cohorts 1930 and 1920. For each cohort and gender, the parameters satisfy the conditions for biological reasonableness as in [9].

\begin{tabular}{|l|c|c|c|c|}
\hline & \multicolumn{2}{|c|}{ Cohort 1950, initial age $x=35$} & Cohort 1940, initial age $x=45$ \\
\hline \hline & Female & Male & Female & Male \\
\hline$\lambda_{x}(0)$ & 0.00075028 & 0.00112463 & 0.00207816 & 0.00329542 \\
\hline$\mu_{x}$ & 0.08001563 & 0.08171875 & 0.07561318 & 0.07731571 \\
\hline$\sigma_{x}$ & 0.00010305 & 0.00011789 & 0.00011809 & 0.00012212 \\
\hline Calibration Error & 0.00000006 & 0.00000007 & 0.00000003 & 0.00000046 \\
\hline
\end{tabular}

Table 1: Calibrated values and errors for males and females of cohort 1950 (initial age 35) and 1940 (initial age 45).

\begin{tabular}{|l|c|c|c|c|}
\hline & \multicolumn{2}{|c|}{ Cohort 1930, initial age $x=55$} & Cohort 1920, initial age $x=65$ \\
\hline \hline & Female & Male & Female & Male \\
\hline$\lambda_{x}(0)$ & 0.00588629 & 0.00976351 & 0.016477 & 0.0313689 \\
\hline$\mu_{x}$ & 0.07452112 & 0.07609306 & 0.080625 & 0.06820313 \\
\hline$\sigma_{x}$ & 0.00011364 & 0.00012183 & 0.00009711 & 0.00009953 \\
\hline Calibration Error & 0.00000085 & 0.00000111 & 0.00001412 & 0.00001689 \\
\hline
\end{tabular}

Table 2: Calibrated values and errors for males and females of cohort 1930 (initial age 55) and 1920 (initial age 65).

\subsection{The fair $\xi^{*}$}

\subsubsection{Fixed $\rho$}

For conciseness reasons, we have computed the fair $\xi^{*}$ only for two cohorts for each of the products. We have computed the fair $\xi^{*}$ values for the pure endowment, the term insurance and the endowment insurance for cohorts 1950 and 1940. For these three products we have 
considered two different contract durations: $T=30$ and $T=40$ years. For the immediate lifetime annuity, we have computed the fair $\xi^{*}$ values for cohorts 1930 and 1920 . Common assumptions on the portfolios are $\rho=0.95$ and $r=0.03$. We have considered different portfolio compositions by setting a fixed number of females $n=50$ and varying the number of males $m$ in the range [10,200]. Therefore, the proportion $w=m /(m+n)$ of males in the portfolio varies from a minimum of $17 \%$ to a maximum of $80 \%$. Tables 3 and 4 report the fair $\xi^{*}$ for pure endowment (PE), term insurance (TI), and endowment insurance (EI) for $T=30,40$ for cohorts 1950 (initial age 35) and 1940 (initial age 45). Table 5 reports the fair $\xi^{*}$ for the immediate lifetime annuity (ILA) for cohorts 1930 (initial age 55), and 1920 (initial age 65), respectively. In each table the second column reports the proportion $w=m /(m+n)$ of males in the portfolio.

\begin{tabular}{|l|c||c|c||c|c||c|c|}
\hline$m$ & $w=\frac{m}{m+n}$ & PE, $T=30$ & PE, $T=40$ & TI, $T=30$ & TI, $T=40$ & EI, $T=30$ & EI, $T=40$ \\
\hline 10 & 0.167 & 0.1629 & 0.1577 & 0.1635 & 0.1593 & 0.1649 & 0.1627 \\
20 & 0.286 & 0.2802 & 0.2724 & 0.2810 & 0.2748 & 0.2831 & 0.2780 \\
50 & 0.500 & 0.4932 & 0.4834 & 0.4942 & 0.4865 & 0.4968 & 0.4929 \\
100 & 0.667 & 0.6606 & 0.6517 & 0.6614 & 0.6545 & 0.6638 & 0.6603 \\
150 & 0.750 & 0.7449 & 0.7373 & 0.7436 & 0.7397 & 0.7496 & 0.7447 \\
200 & 0.800 & 0.7959 & 0.7899 & 0.7962 & 0.7912 & 0.7979 & 0.7954 \\
\hline
\end{tabular}

Table 3: Fair $\xi^{*}$ for pure endowment (PE), term insurance (TI) and endowment insurance (EI) with parameters: $n=50, \rho=0.95, r=0.03$, generation born in 1950, initial age 35 .

We observe the following:

- $\xi^{*}$, that is the weight given to the males' mortality intensity in the fair unisex intensity, is never equal to the fraction of males in the portfolio $w=m /(m+n)$; this means that the mix of the mortality intensities of males and females cannot be done using exactly the volume-related weights;

- nonetheless, $\xi^{*}$ strongly depends on $w$ and increases when $w=m /(m+n)$ increases; this is obvious, because when there are more males in the portfolio, more weight must be given to the males' mortality; 


\begin{tabular}{|l|c||c|c||c|c||c|c|}
\hline$m$ & $w=\frac{m}{m+n}$ & PE, $T=30$ & PE, $T=40$ & TI, $T=30$ & TI, $T=40$ & EI, $T=30$ & EI, $T=40$ \\
\hline 10 & 0.167 & 0.1564 & 0.1436 & 0.1579 & 0.1485 & 0.1618 & 0.1569 \\
20 & 0.286 & 0.2704 & 0.2509 & 0.2727 & 0.2586 & 0.2785 & 0.2712 \\
50 & 0.500 & 0.4808 & 0.4553 & 0.4838 & 0.4655 & 0.4911 & 0.4820 \\
100 & 0.667 & 0.6493 & 0.6253 & 0.6521 & 0.6352 & 0.6587 & 0.6504 \\
150 & 0.750 & 0.7353 & 0.7143 & 0.7376 & 0.7230 & 0.7433 & 0.7362 \\
200 & 0.800 & 0.7873 & 0.7681 & 0.7893 & 0.7768 & 0.7942 & 0.7882 \\
\hline
\end{tabular}

Table 4: Fair $\xi^{*}$ for pure endowment (PE), term insurance (TI) and endowment insurance (EI) with parameters: $n=50, \rho=0.95, r=0.03$, generation born in 1940, initial age 45 .

\begin{tabular}{|l|c|c|c|}
\hline$m$ & $w=\frac{m}{m+n}$ & ILA $(x=55)$ & ILA $(x=65)$ \\
\hline 10 & 0.167 & 0.1345 & 0.1391 \\
20 & 0.286 & 0.2375 & 0.2442 \\
50 & 0.500 & 0.4389 & 0.4468 \\
100 & 0.667 & 0.6109 & 0.6176 \\
150 & 0.750 & 0.7023 & 0.7079 \\
200 & 0.800 & 0.7590 & 0.7636 \\
\hline
\end{tabular}

Table 5: Fair $\xi^{*}$ for immediate lifetime annuity (ILA) with parameters for generation born in $1930(x=55)$ and in $1920(x=65): n=50, \rho=0.95, r=0.03, \omega=120$.

- for all life insurance products considered, all cohorts and durations, $\xi^{*}$ is smaller than $w=m /(m+n)$; for the pure endowment, this is in line with the result of Proposition 5.2 , noting that the calibrated values of the volatilities $\sigma_{m}$ and $\sigma_{f}$ are small, and therefore for both genders the stochastic intensity is not significantly different from a deterministic force of mortality. This result sheds light on the complex relationship between $\xi^{*}$ and $w$, and points out that the fair $\xi^{*}$ equivalent to a given males' proportion $w$ is smaller than $w$, or, in other words $\xi^{*}(w) \leq w$;

- for each cohort, portfolio size and duration, $\xi^{*}$ is greater for the term insurance than for the pure endowment; this is reasonable, because with the term insurance it is prudent to give more weight to the males' mortality and with the pure endowment it is prudent to give more weight to the females' mortality; this is consistent with the 
current practice of giving more weight to the males' life table for the term insurance and less weight to the males' life table for the pure endowment;

- for each cohort, portfolio size and duration, $\xi^{*}$ is greater for the endowment insurance than for the pure endowment and the term insurance. This can be explained by noting that the actuarial value of a standard endowment is equal to the price of a zero coupon bond plus the premium of a term insurance with decreasing sum assured (see e.g. [11]), therefore from the probabilistic point of view the endowment is similar to the term insurance;

- immediate lifetime annuities deliver plausible and intuitive results: $\xi^{*}$ is always substantially smaller than $w=m /(m+n)$, i.e. always more weight must be given to the females' mortality. The difference between $w$ and $\xi^{*}$ turns out to be higher with annuities than with pure endowment (although different cohorts are considered for the two products): this can be explained by noting that the lifetime annuity is paid until death of the policyholder; at very old ages the portfolio is likely to consist mainly of females, and therefore in a portfolio of annuities the mortality of females should be dominating those of males; this requirement is crucial for lifetime annuities but is less important for pure endowment, given that towards the time duration $T$ the portfolio of pure endowment is not likely to consists mainly of females;

- overall, $\xi^{*}$ is higher for shorter duration $(T=30)$ and smaller for longer duration $(T=40)$; this result is meaningful and indicates that when the duration is high, the importance given to females should increase; this is probably due to the fact that females live longer, so the share of males $m /(m+n)$ decreases in the long run, as more males die.

The comparisons between $\xi^{*}$ across different products, cohorts, duration $T$ and males' proportions $w$ highlight that $\xi^{*}$ is affected by all these components, and reflects all information about the future evolution of mortality of the mixed portfolio. These different values shed light on how the unisex mortality should be treated in different portfolios in order to ensure actuarial fairness. 


\subsubsection{Varying $\rho$}

In Tables 3,4 , and 5 , the correlation coefficient $\rho$ is set to 0.95 . However, $\xi^{*}$ in principle may depend also on $\rho$ and, if this is the case, the insurer should take into account this dependence in pricing unisex tariffs. Thus, we have investigated the dependence of $\xi^{*}$ on $\rho$. Figures $3-5$ show, for the considered cohorts, the effect of $\rho$ on $\xi^{*}$ for the pure endowment, the term insurance, the endowment insurance and the immediate lifetime annuity. Common assumptions on the portfolios are $m=n=50$, that implies $w=m /(m+n)=0.5, T=30$ and $r=0.03$. For the duration $T=40$ we would get similar figures.

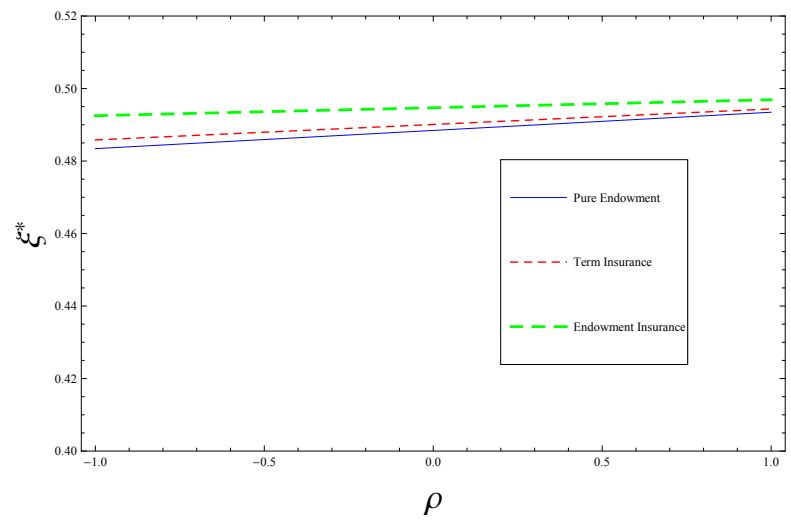

Figure 3. $\xi^{*}$ versus $\rho$, cohort 1950.

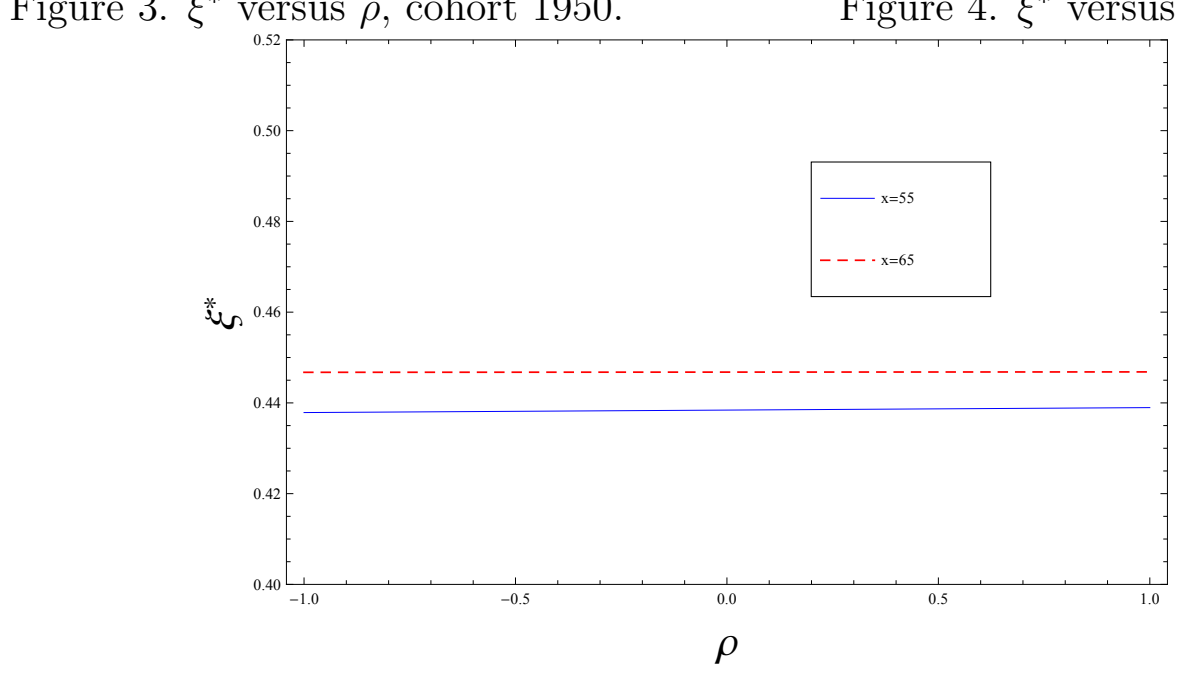

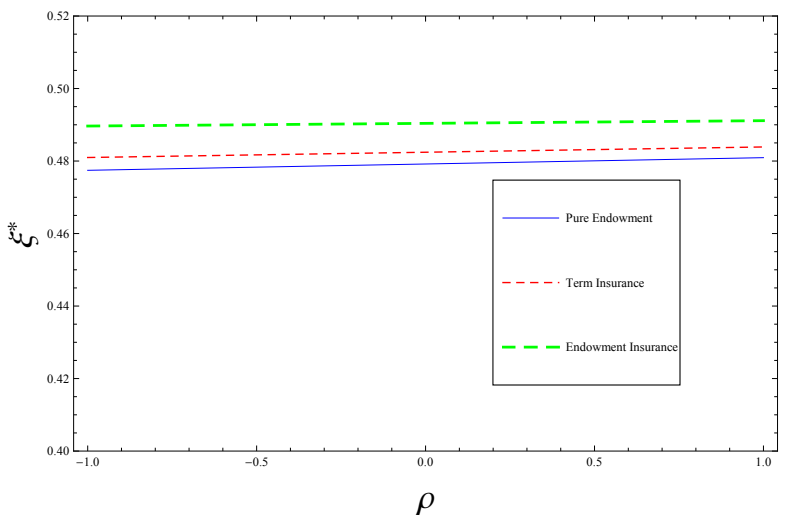

Figure 4. $\xi^{*}$ versus $\rho$, cohort 1940.

Figure 5. $\xi^{*}$ versus $\rho$ for immediate lifetime annuity, cohort $1930(x=55)$ and cohort 1920 $(x=65)$. 
The graphs show that $\xi^{*}$ is almost constant when $\rho$ varies for cohorts 1920, 1930 and 1940 , and is slightly increasing for cohort 1950. Therefore, the effect of $\rho$ on $\xi^{*}$ is essentially negligible. This is consistent with related work, not reported here, that shows that the calculation of the fair parameters $\xi^{*}$ with the sample correlation $\rho^{\text {sample }}$ delivers numerical results that are identical (or almost identical) to those presented in the previous section.

\section{$7 \quad$ Periodic premiums}

In Section 6.2.1 we have calculated $\xi^{*}$ only in the case of single premium. In this section, we consider the case of periodic annual premiums, and calculate the corresponding fair mortality intensity parameter. We note that relaxing the single premium assumption makes the fair mortality intensity mixing parameter look less intuitive and more complex, even for simple contracts like pure endowment. But theoretically, it is possible to track down the equations that can be solved for the fair mortality intensity mixing parameter. Taking the pure endowment with unit sum assured as an example, let us elaborate on this issue. Letting $\pi^{m}, \pi^{f}$ and $\pi^{u}$ denote the fair flat periodic premiums, they satisfy:

$$
\begin{aligned}
\sum_{k=0}^{T-1} S^{i}(0, k) \pi^{i} e^{-r k} & =e^{-r T} S^{i}(0, T) \\
\Rightarrow \pi^{i} & =\frac{e^{-r T} S^{i}(0, T)}{\sum_{k=0}^{T-1} S^{i}(0, k) e^{-r k}} \quad i=m, f, u
\end{aligned}
$$

The fair mortality intensity parameter $\xi^{*}$ results from solving the following equality:

$$
\frac{e^{-r T} S^{u}(0, T)}{\sum_{k=0}^{T-1} S^{u}(0, k) e^{-r k}}=w \frac{e^{-r T} S^{m}(0, T)}{\sum_{k=0}^{T-1} S^{m}(0, k) e^{-r k}}+(1-w) \frac{e^{-r T} S^{f}(0, T)}{\sum_{k=0}^{T-1} S^{f}(0, k) e^{-r k}},
$$

where $\xi^{*}$ is hidden in $S^{u}(0, k), k=1, \cdots, T$. Compared to the single premium case, $\xi^{*}$ depends also on the interest rate $r$. Similar equations hold for the other life insurance products, and we do not report them for conciseness reasons.

We have calculated the fair $\xi^{*}$ in the presence of periodic annual premiums payable over the whole policy duration for the term insurance, the pure endowment and the endowment (we 
have not done it for lifetime annuities, because in that case the single premium is usually adopted). The results are reported in Tables 6 and 7 .

\begin{tabular}{|l|c||c|c||c|c||c|c|}
\hline$m$ & $w=\frac{m}{m+n}$ & PE, $T=30$ & PE, $T=40$ & TI, $T=30$ & TI, $T=40$ & EI, $T=30$ & EI, $T=40$ \\
\hline 10 & 0.167 & 0.1636 & 0.1587 & 0.1649 & 0.1617 & 0.1664 & 0.1653 \\
20 & 0.286 & 0.2813 & 0.2740 & 0.2830 & 0.2784 & 0.2853 & 0.2837 \\
50 & 0.500 & 0.4945 & 0.4854 & 0.4967 & 0.4909 & 0.4995 & 0.4875 \\
100 & 0.667 & 0.6618 & 0.6535 & 0.6637 & 0.6585 & 0.6662 & 0.6644 \\
150 & 0.750 & 0.7459 & 0.7388 & 0.7475 & 0.7431 & 0.7496 & 0.7481 \\
200 & 0.800 & 0.7965 & 0.7904 & 0.7979 & 0.7941 & 0.7996 & 0.7984 \\
\hline
\end{tabular}

Table 6: Fair $\xi^{*}$ in case of periodic premiums for pure endowment (PE), term insurance (TI) and endowment insurance (EI) with parameters: $n=50, \rho=0.855, r=0.03$, generation born in 1950, initial age 35 .

\begin{tabular}{|l|c||c|c||c|c||c|c|}
\hline$m$ & $w=\frac{m}{m+n}$ & PE, $T=30$ & PE, $T=40$ & TI, $T=30$ & TI, $T=40$ & EI, $T=30$ & EI, $T=40$ \\
\hline 10 & 0.167 & 0.1591 & 0.1476 & 0.1626 & 0.1562 & 0.1663 & 0.1640 \\
20 & 0.286 & 0.2745 & 0.2570 & 0.2797 & 0.2702 & 0.2852 & 0.2819 \\
50 & 0.500 & 0.4861 & 0.4663 & 0.4926 & 0.4807 & 0.4994 & 0.4952 \\
100 & 0.667 & 0.6541 & 0.6329 & 0.6600 & 0.6493 & 0.6661 & 0.6624 \\
150 & 0.750 & 0.7394 & 0.7210 & 0.7444 & 0.7352 & 0.7495 & 0.7464 \\
200 & 0.800 & 0.7909 & 0.7750 & 0.7952 & 0.7874 & 0.7996 & 0.7969 \\
\hline
\end{tabular}

Table 7: Fair $\xi^{*}$ in case of periodic premiums for pure endowment (PE), term insurance (TI) and endowment insurance (EI) with parameters: $n=50, \rho=0.832, r=0.03$, generation born in 1940, initial age 45 .

The effects demonstrated in the single premium case can be observed here, too. The exclusive difference in this case is that the magnitude of $\xi^{*}$ here is higher than that in the single premium case. In other words, by charging periodic premiums, we obtain $\xi^{*}$ closer to the portfolio weight $w$. In particular, for endowment insurance contracts, $\xi^{*}$ can become extremely close to $w$, if the number of males is large in the portfolio. 


\section{Time-varying $\xi^{*}$}

In Section 5, we have introduced the unisex fair mortality intensity mixing parameter $\xi^{*}$ in the case of the single premium as the parameter that ensures actuarial fairness at time 0 only. In Section 7, we have dealt with the case of periodic premiums, but considering again the actuarial fairness at time 0 only. It is clear that if at any time $t>0$ we define a unisex mortality model for the portfolio by using the fair parameter $\xi^{*}$ calculated at time 0 , the resulting unisex mortality model will no longer be fair, because from 0 to $t$ the proportion of males in the portfolio does not remain constant trough time, due to the different mortality paths of males and female. It might however be interesting to calculate the fair mortality mixing parameter $\xi_{t}^{*}$ at time $t$ that is equivalent to the updated males' proportion at time $t, w_{t}$. This could be useful if, for instance, the insurer prefers to do reserving on the whole portfolio rather than on the two gender-based subportfolios and needs a unisex fair mortality model. ${ }^{5}$ Therefore, in this section we address the issue of time-varying $\xi_{t}^{*}$.

The calculation of the fair $\xi_{t}^{*}$ at time $t$ is not straightforward. Taking a pure endowment insurance contract as an example, the initial fairness results from satisfying the following condition:

$$
m S^{m}(0, T)+n S^{f}(0, T)=(m+n) S^{u}(0, T) .
$$

If we want to compute the fair $\xi_{t}^{*}$ for a later time point $t \in(0, T)$, we need to know how many policyholders have survived time $t$, and the realized mortality intensities. All these are unknown at time 0 and can be simulated. But if we use simulated values, we will obtain a different $\xi_{t}^{*}$ for every different simulated number, which make a comparison with the initial $\xi^{*}$ difficult. To overcome this problem, we use the expected survival number until time $t$ as the realized number of survivals, i.e. $w_{t}=m S^{m}(0, T) /\left(m S^{m}(0, T)+n S^{f}(0, T)\right)$. Furthermore, we assume that the realized mortality intensities at time $t$ equals their expected value, i.e.

\footnotetext{
${ }^{5}$ This could be the case of Danish insurers, considering that after the EU Gender Directive in Denmark regulators have introduced fiscal incentives to do reserving considering the whole portfolio rather than the two subportfolios of males and females. Therefore, the knowledge of a single unisex mortality model that respects actuarial fairness at any time can be useful in all countries where such fiscal incentives were introduced.
} 
$E\left(\lambda^{i}(t)\right)=\lambda^{i}(0) e^{\mu^{i} t}$. In other words, we assume that the fair $\xi_{t}^{*}$ satisfies:

$$
m S^{m}(0, t) S^{m}(0, t, T)+n S^{f}(0, t) S^{f}(0, t, T)=\left(m S^{m}(0, t)+n S^{f}(0, t)\right) S^{u}(0, t, T),
$$

here we have used $S^{i}(0, t, T), i=m, f, u$ to point out the fact that we are using the expected value in 0 of $\lambda^{i}(t), E\left(\lambda^{i}(t)\right)$, to compute the survival probabilities during $[t, T]$.

Figures 6, 8, 10, 12 illustrate how $\xi_{t}^{*}$ change in time for the four contract types: pure endowment, term insurance, endowment insurance and immediate lifetime annuities. Figure 7, 9,11 and 13 report the difference $w_{t}-\xi_{t}^{*}$ over time, that is crucial given that $\xi_{t}^{*}$ depends essentially on $w_{t}$. Here we have used $m=n=50$ and the sample correlation coefficients. ${ }^{6}$ Note that in Figure $12, \xi_{t}^{*}$ is only plotted until $t=50$ for generation 1920.

We observe the following:

- In the figures, we can distinguish two distinct patterns for $\xi_{t}^{*}$ and the difference $w_{t}-\xi_{t}^{*}$ : one is for the three products PE, TI, and EI, the other is for ILA. In other words, products with a time duration $T$ display strong similarities, that make them different from lifetime annuities.

- For all the contract types, $w_{t}>\xi_{t}^{*}$ for all $t$. Interestingly, when $t$ approaches the contract maturity (for the lifetime annuity, the maximal age), the difference $w_{t}-\xi_{t}^{*}$ becomes smaller and tends to 0 . In other words, when maturity approaches the fair $\xi_{t}^{*}$ becomes closer and closer to the real portfolio weight $w_{t}$.

- For PE, TI, and EI, $\xi_{t}^{*}$ is almost constant for the youngest cohort 1950, while it is slightly decreasing for cohort 1940 .

- For PE, TI, and EI, the positive difference $w_{t}-\xi_{t}^{*}$ is lower for the youngest cohort 1950 than for the oldest cohort 1940.

- For ILA, $\xi_{t}^{*}$ sharply decreases towards 0 as time goes by for cohort 1930. This is due to the fact that at very old ages only females remain in the pool and this pushes down both $w_{t}$ and $\xi_{t}^{*}$. For cohort $1920, \xi_{t}^{*}$ decreases towards approximately 0.1 as the

\footnotetext{
${ }^{6}$ The sample correlation $\rho$ is 0.855 for cohort $1950,0.832$ for cohort $1940,0.876$ for cohort 1930 and 0.948 for cohort 1920 .
} 
maximal age $\omega$ approaches. Considering that also for this cohort $w_{t}-\xi_{t}^{*}$ tends to 0 when the age tends to $\omega$, this means that also $w_{t}$ tends approximately to 0.1 .

- For ILA, the difference $w_{t}-\xi_{t}^{*}$ for cohort 1930 slightly increases for 30 years, then drops quickly towards 0 close to maximal age. For cohort 1920 there is a gradual reduction towards 0 when maximal age approaches.

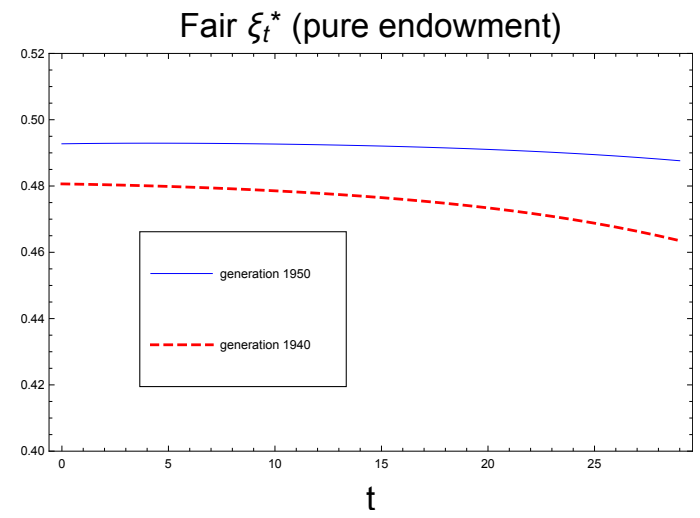

Figure 6. Time-varying fair $\xi_{t}^{*}$

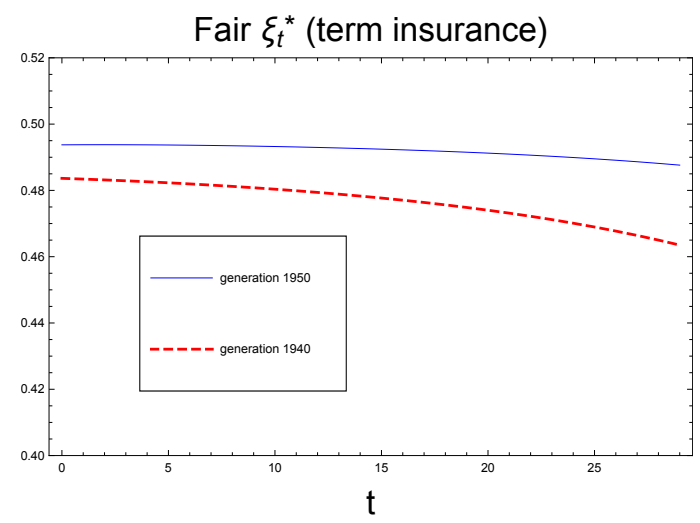

Figure 8. Time-varying fair $\xi_{t}^{*}$

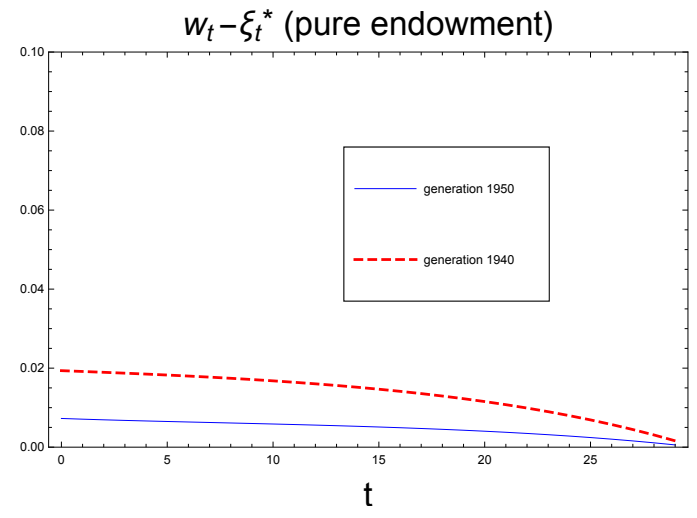

Figure 7. Difference of $w_{t}$ and $\xi_{t}^{*}$

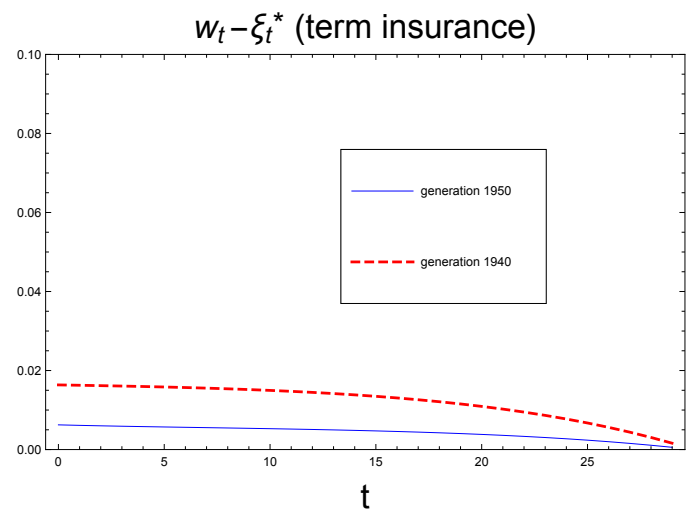

Figure 9. Difference of $w_{t}$ and $\xi_{t}^{*}$ 


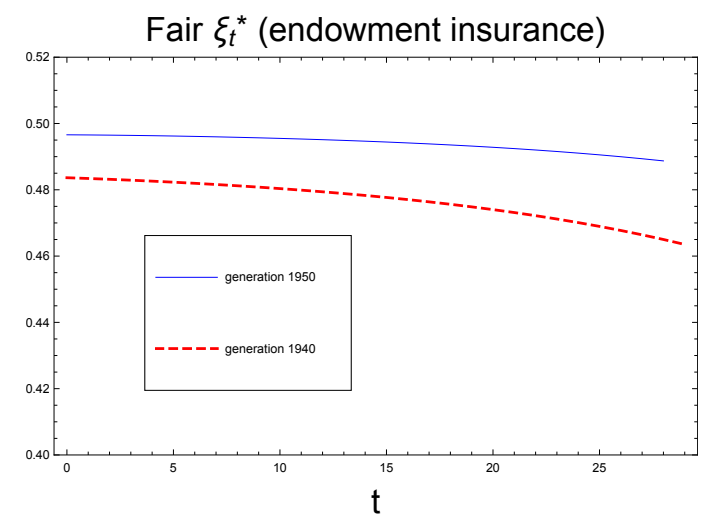

Figure 10. Time-varying fair $\xi_{t}^{*}$

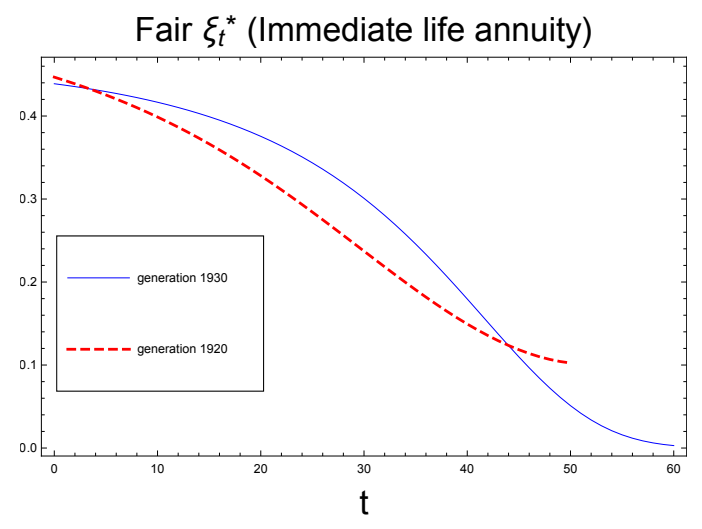

Figure 12. Time-varying fair $\xi_{t}^{*}$

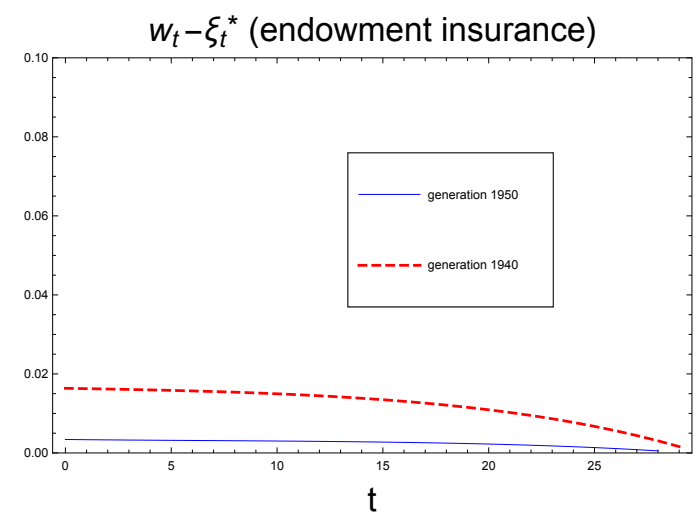

Figure 11. Difference of $w_{t}$ and $\xi_{t}^{*}$

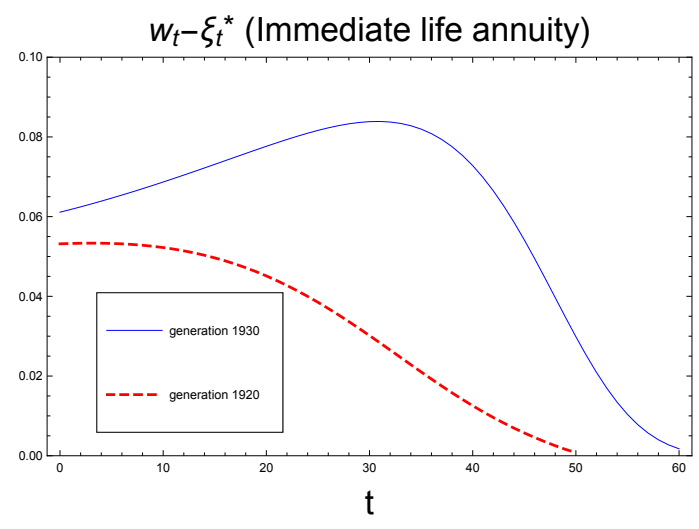

Figure 13. Difference of $w_{t}$ and $\xi_{t}^{*}$

\section{Concluding remarks}

The EU Gender Directive (European Union Directive 2004/113/EC) has introduced binding rules for the pricing of life insurance products. In the lack of theoretical models, the current practice adopted by actuaries for the calculation of the unisex premium seems to be a weighted average of the gender-specific life tables, with proper corrections linked to the type of insurance product. This paper proposes a theoretical model for the pricing of the unisex premium of a life insurance products. First, we recall the notion of unisex actuarial fairness principle and the corresponding unisex actuarially fair premium. Second, as the 
main contribution of the paper, we introduce the fair unisex mortality intensity, that is the unisex intensity underlying the unisex fair premium.

The numerical application is rich of interesting results. First, the comparison between the unisex fair mortality models to be adopted with the same proportion males/females in the portfolio for different insurance products provides meaningful and useful information. Indeed, with the same males/females proportion, the weight to be given to the males' mortality in the fair unisex mortality model is different for different insurance product. For instance, if the insurer has two portfolios, one with term insurances (or endowments) and the other with lifetime annuities (or pure endowments), which have the same proportion males/females, the weight that he must give to the males' mortality intensity in the portfolio of term insurances (or endowments) is higher than that to be given in the portfolio of lifetime annuities (or pure endowments). This is also prudent and consistent with current actuarial practice. Second, as a feature of the mortality model selected (stochastic force of mortality) we find that the weight to be given to the males' mortality in the unisex model is smaller than the proportion of males in the portfolio. Third, by setting the actuarial fairness principle on an annual basis rather than at the policy issue only, we find that when the time horizon of the policy approaches the weight to be given to the males' mortality converges to the proportion of males in the portfolio. Annual fairness condition and time-varying unisex mortality models could be useful if, based on the EU Gender Directive, regulators were to encourage (or require) the reserving done on the whole portfolio basis rather than on gender-based subportfolios as it is happening in some countries.

In the present paper, we apply the traditional actuarial equivalence principle at the portfolio level in a modern way and address the specific recent problem caused by mandatory unisex tariff. The unisex fair premium as well as the fair unisex mortality intensity could be useful tools for the life insurance company. The knowledge of the fair unisex mortality model should enhance the understanding of the nature of the risk of a mixed portfolio. The use of the present model for the calculation of Solvency II SCR for mixed life insurance portfolios is in the agenda for future research. 
Finally, this paper leaves space for further research. Possible extensions are: the introduction of a stochastic discount factor; the introduction of safety loading and commissions; a stochastic proportion of males in the portfolio; the derivation of a unisex fair mortality model underlying the fair unisex premium with a different mortality model.

\section{References}

[1] Biffis, E. (2005). Affine processes for dynamic mortality and actuarial valuations, Insurance: Mathematics and Economics 37, 443-468.

[2] Dahl, M. (2004). Stochastic mortality in life insurance: market reserves and mortalitylinked insurance contracts, Insurance: Mathematics and Economics 35, 113-136.

[3] Duffie, D., Pan, J. and Singleton, K. (2000). Transformation Analysis and Asset Pricing for Affine Jump Diffussion, Econometrica 68(6), 1343-1376.

[4] Ordine degli Attuari (2012). Gender Directive - Linee Guida Vita e Previdenza Complementare, http://www.ordineattuari.it/linee-guida/linea-guida-gender-directive/.

[5] Guillén, M (2012). Sexless and beautiful data: from quantity to quality. Annals of Actuarial Science 6, 231-234.

[6] Ornelas, A. and Guillén, M. (2013). A Comparison between General Population Mortality and Life Tables for Insurance in Mexico under Gender Proportion Inequality. Revista de metodos cuantitativos para la economia y la empresa 16, 47-67.

[7] Luciano, E., Regis, L. and Vigna, E. (2012). Delta and Gamma hedging of mortality and interest rate risk, Insurance: Mathematics and Economics 50, 402-412.

[8] Luciano, E., Regis, L. and Vigna, E. (2015). Single and cross-generation natural hedging of longevity and financial risk, to appear on Journal of Risk and Insurance.

[9] Luciano, E. and Vigna, E. (2008). Mortality Risk via Affine Stochastic Intensities: Calibration and Empirical Relevance, Belgian Actuarial Bulletin 8(1), 5-16. 
[10] Milevsky, M. and Promislow, S. D. (2001). Mortality derivatives and the option to annuitise, Insurance: Mathematics and Economics 29, 299-318.

[11] Pitacco, E. and Olivieri, A (2011). Introduction to Insurance Mathematics, SpingerVerlag Berlin Heidelberg.

[12] Sass, J. and Seifried, F.T. (2014). Insurance markets and unisex tariffs: is the European Court of Justice improving or destroying welfare? Scandinavian Actuarial Journal 3, 228-254,

[13] Schmeiser, H., Störmer, T., and Wagner, J. (2014). Unisex Insurance Pricing: Consumers' Perception and Market Implications, The Geneva Papers 39, 322-350.

[14] Schrager, D. (2006). Affine stochastic mortality, Insurance: Mathematics and Economics $38,81-97$.

[15] Thiery, Y. and Van Schoubroeck, C. (2006), Fairness and equality in insurance classification, The Geneva Papers 31, 190-211. 